\title{
Heparin-induced thrombocytopenia: Pathophysiology and new treatment options
}

\author{
Harenberg J., Jörg I., Fenyvesi T. \\ 4th Dept. of Medicine, University Hospital Mannheim, Ruprecht-Karls University Heidelberg, Mannheim, Germany
}

\section{Key Words}

Heparin-induced thrombocytopenia, danaparoid, rhirudin, bivalirudin, argatroban, dextransulfate, dermatansulfate, fondaparinux, ximelagatran

\begin{abstract}
Heparin induced thrombocytopenia (HIT) is a severe complication of heparin therapy. It is generally accompanied by a paradoxical decrease in platelets leading to activation of platelets and of the coagulation system. HIT type I is a mild, transient, non-immune disorder. HIT type II is an immune-mediated reaction towards neo-antigen on PF4, which is platelet factor 4 (PF4) that is exposed upon binding to heparins. A low sulfated octasaccharide is required for binding to PF4. The generated immunoglobulines bridge platelets by binding to the FcgRllareceptor. In patients with HIT type II heparin /LMWheparin has to be discontinued immediately upon clinical suspicion. Diagnosis can be confirmed by laboratory tests. As patients are at high risk for or because they have developed thromboembolism, anticoagulation is mandatory, despite thrombocytopenia. Treatment options are danaparoid, r-hirudin, bivalirudin, argatroban, dextransulfate, and dermatansulfate. In future, fondaparinux and ximelagatran may be considered for treatment.
\end{abstract}

\section{Introduction}

Prophylaxis and treatment of thromboembolism is performed for over half a century with unfractionated heparin and since more than 20 years with low-molecular weight (LMW) heparins. All heparins are linear negatively charged polydisperse mixtures of glycosaminoglycans binding tightly to antithrombin and mediating anticoagulant effects. They do also bind to a number of other proteins in plasma and on endothelium, leukocytes and other cell surfaces. The binding patterns change upon activation of blood coagulation and cells except for antithrombin. Unique oligosaccharide sequences of heparin have been identified to bind some of these proteins. A pentasaccharide binds tightly to antithrombin and an octasaccharide unit to platelet factor 4 (PF4), respectively. The saccharide sequence does overlap in the heparin chain. After binding of heparins to antithrombin and PF4, conformational changes of the proteins occur [1].

The analysis of the binding of the saccharide sequences to antithrombin and PF4 are of special interest because they induce opposite clinical effect. Binding of heparins to antithrombin induces the anticoagulant effect by binding to activated clotting factors and thrombin activated fibrinolysis inhibitor and by releasing tissue factor pathway inhibitor. In contrast, the conformational change of PF4 by binding to heparins induces a neoepitope, which may become antigenic producing specific antibodies in general of the $\mathrm{IgG}$ subclass. The macromolecular antibody-heparin-PF4 complex binds to Fc-gamma RIIa receptor of platelets and induces activation

\section{KARGER}

Fax +4161306 1234
Job Harenberg, M.D.

Professor of Medicine - IV. Dept. of Medicine - University Hospital Mannheim

Ruprecht-Karls University Heidelberg - Theodor-Kutzer-Ufer

D-68167 Mannheim, Germany

Tel: +49/621/383-3378, -2789 - Fax: +49/621/383-3808

Email: j-harenberg@t-online.de 
and aggregation of them by bridging. These mechanisms lead to the controversial effects of hypo- and hypercoagulability of heparins. This overview focuses on the second feature of heparin's activity.

\section{Definition of HIT type I and II}

HIT type I is associated with a transient thrombocytopenia during a few days of heparin therapy. Platelets decrease up to $50 \%$ of the initial value. They may increase despite continuation of heparin therapy. Other features are not observed in HIT type I disease.

HIT type II is an immune mediated disease during heparin therapy. Thrombocytopenia occurs in general between days 5 and 15 after the onset of heparin therapy. Onset of thrombocytopenia may occur earlier after re-exposition and later in some cases. Platelets drop to values of $<100,000 \times 10^{9} / 1$ or decrease by more than $50 \%$ of the pretreatment value. Arterial or venous thromboembolism or cutaneous necrosis [2] may develop, and antibodies to the heparin-PF4 complex may appear. Other causes of thrombocytopenia have to be excluded. Platelet count normalizes only after withdrawal of heparin. If patients with HIT-type II receive heparin again, the disease reoccurs. Re-exposition to any heparin is contraindicated. Two of the 3 pathologies of thrombocytopenia, thromboembolism or heparin/PF4 antibodies confirm the diagnosis.

\section{Pathophysiology of HIT}

The pathophysiology of HIT type I is considered to be induced by an interaction of heparin with platelets resulting in a thrombocytopenia within few days. Low concentrations of circulating PF4 to heparin seem to be required to bind on the platelet surface. It is unlikely that heparin alone induces platelet aggregation on the surface of silent, not activated platelets. The precise mechanism remains unknown, by which only higher PF4 concentrations should induce the neoantigenic transformation of PF4 in HIT type II. The lack of an immunologic mechanism is supported by the observation that platelet count increases in some patients despite continuation of heparin. Risk factors for HIT-type I are not described. The incidence of HIT type I ranges from $0.5 \%$ up to $3 \%$ in the literature.

The pathophysiology of HIT type II is a complex immune mediated mechanism. It is initiated by binding of an octasaccharide of exogenous heparin to PF4. The binding of heparin to PF4 decreases with lowering the molecular weight of heparins, the octasaccharide being the shortest unit. It is uncertain, whether the number of specific octasaccharides is present once in a heparin molecule or whether the number decreases lowering the molecular weight of heparin. If only one octasac- charide sequence per heparin is present the differences between heparin and LMW heparin suggest a second binding site of heparin on PF4.

Binding of endogenous octasaccharide sequences in the heparansulfates located on the endothelium may also be involved. PF4 undergoes a conformational change binding to heparin and by this presents a neoepitope. The neoepitope may become antigenic. The immune response is polyclonal and polyspecific. The generated HIT antibodies are of the IgG class in general, but also IgM and IgA-HIT antibodies are generated. Thus, HIT antibodies [3] recognize more than one neoepitope on PF4. Site 1 of the neoepitope involves the domain between the third and fourth cysteine residue in PF4. The site 2 epitope is located between the $\mathrm{N}$-terminus and the prolin aminoacid 34 of PF4. It lies adjectant to site 1 in the crystal structure of the PF4 tetramer [3].

The IgG antibodies cross-link the platelet Fc-gamma-RIIa receptors of two platelets resulting in platelet activation independent from the glycoprotein IIb/IIIa complex. The receptor carries a functionally relevant polymorphism, of which the wild type is Fc-gamma-RIIa-R-H-131. In HIT-type II patients the Fc-gamma-RIIa-R/R131 allele was over-represented if patients develop thromboembolism [4]. The HIT-IgG antibodies also bind to PF4 located at the heparansulfate of endothelial cells. Blood coagulation is initiated both by activated platelets and by activation through the endothelium.

HIT type II patients are characterized by clinical risk factors for developing thromboembolism leading to elevated concentrations of PF4 in contrast to HIT type I. The risk factor include all those which lead to an elevated risk for development of venous thromboembolism.

\section{Laboratory tests for HIT antibodies}

The diagnosis of HIT may be difficult on the basis of clinical symptoms alone, especially in patients with other diseases, which may induce thrombocytopenia. Laboratory confirmation of HIT is therefore required using biological or antigen assays. The most precise assay for confirmation of HIT-IgG antibodies is based on the release of serotonin from donor platelets in the presence of patients serum (containing the pathogenic $\mathrm{IgG})$ and a low concentration of heparin $(0.1 \mathrm{IU}$ per ml per 100,000 platelets per microliter). Release of serotonin using $\mathrm{H} 3$ serotonin, and quantification of released serotonin by an ELISA and a HPLC method detect HIT-IgG antibodies with a sensitivity and specificity between $95 \%$ and $98 \%$ each $[5,6,7]$.

The impact of the type of heparin, the patient population on the risk for HIT and the assay (biologic or antigenic) consistently influence the observed frequency of HIT-type II. Among cardiac patients up to $20 \%$ HIT-IgG antibody formation was observed, followed by orthopedic surgery in $9 \%$ of patients 
treated with heparin and in $3 \%$ of these patients treated with LMW heparin. The serotonin release assay was performed in these patients. The clinical diagnosis of HIT-type II was made in $1 \%, 5 \%$ and $1 \%$ in the three groups of patients, respectively. Thus, there is evidence that the time course of HIT-IgG antibodies and the pathogenicity of the antibodies may be relevant for the HIT-type II disease [8].

The results of antigenic HIT-IgG antibody assays give higher values for the development of antibodies compared to the serotonin release assay. Among cardiac patients up to 50\% HIT-IgG antibody formation was observed, followed by orthopedic surgery in $14 \%$ of patients treated with heparin and in $7.5 \%$ of these patients treated with LMW heparin. In patients suffering from acute myocardial infarction, antibodies were detected despite less of exposure to heparin. Patients with renal dialysis HIT antibodies developed in $45 \%$ HIT-IgG antibodies without a relation to a decrease of platelets or to development of fibrin deposits on dialysis membranes or thrombotic events. In about $15 \%$ of HIT cases, other proteins such as NAP-2 and IL-8 might be involved in forming the neoantigen [9].

\section{Time course of HIT-antibodies and disease}

The generation of HIT antibodies may take a certain time period to exposure to heparin. They also may appear after termination of heparin therapy within a couple of days [10, 11]. The combination of two specific test systems has been found to improve the sensitivity and specificity of the detection of HIT antibodies in patients with HIT type II. About $20 \%$ of patients seroconverted from negative results to positive results in an antigenic or biological assay within the subsequent 7 days. Combining the positive results of assays increased the sensitivity of $85 \%$ at day 1 for each assay to $100 \%$ at day 7 combining the positive results of the two assays (Figure 1). Four patterns of generation of HIT antibodies were reported:

Pattern 1: positive antigen and biological assay at the day of clinical diagnosis of HIT type II.

Pattern 2: negative antigen assay and positive biological assay at the time of diagnosis of HIT type II.

Pattern 3: negative biological assay and positive antigen assay at the day of diagnosis of HIT type II

Pattern $4 \mathrm{a}$ and $\mathrm{b}$ : seroconvertion of the antigen or biological assay within 7 days after diagnosis of HIT type II and stopping heparin therapy [12].

IgG antibodies in HIT patients decrease over 40 days. The decrease of antibodies is independent from the increase of platelet count. It is suggested that during this period patients be on high risk to develop HIT type II due to the presence of endogenous glycosaminoglycans/heparinsulfate on the endothelial surface. Therefore, patients developing thrombocytopenia during heparin therapy may develop thrombotic

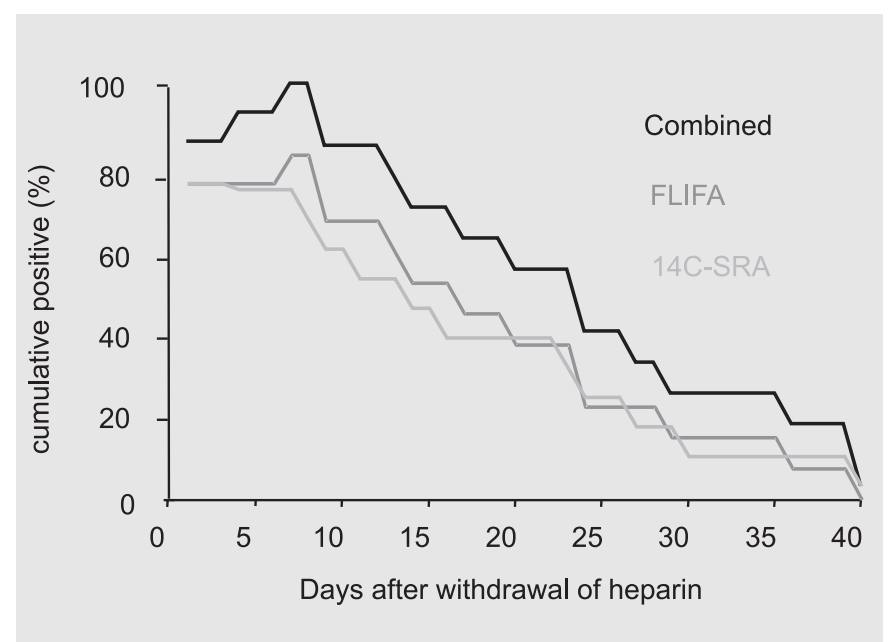

Fig. 1. Combination of positive antigenic (FLIFA, fluorescent linked immunofiltration assay, in 6) for laboratory confirmation of clinical high probability of HIT (with permission from ref. 10)

complication during or after termination of heparin therapy due to the presence of free IgG HIT antibody. Thus, HIT type I may progress to HIT type II and has been described in up to $30 \%$ of patients. These considerations lead to the clinical decision that patients with heparin should be controlled for platelet counts during therapy and 1 week after heparin therapy. Heparin should be switched to an alternative anticoagulant for other 7-14 days, if patients have developed thrombocytopenia and other causes for thrombocytopenia having been excluded.

Accordingly, a delayed onset of HIT type II may occur also after termination of heparins after decrease of platelets or initiation of thrombus formation. This leads to the conclusion, that during heparinisation development of thrombocytopenia and/or thrombosis and/or disseminated intravascular coagulation and/or heparin-induced antibodies may occur also after stopping heparins.

Cessation of heparin is the treatment of choice in HIT type II patients. The time course of the generation and the disappearance of HIT type II IgG, IgM, or IgA antibodies determine the clinical onset and offset of the disease.

\section{Treatment options}

Patients with HIT-type I may continue with heparin for anticoagulation. HIT-antibodies have to be negative and other reasons of thrombocytopenia have to be excluded (Figures 2 and 3 ).

Upon clinical suspicion of HIT type II any heparin has to be stopped immediately. All patients need an alternative anticoagulant regimen due to the thrombotic process. The alternative anticoagulants should not interact with platelet factor 4 to 


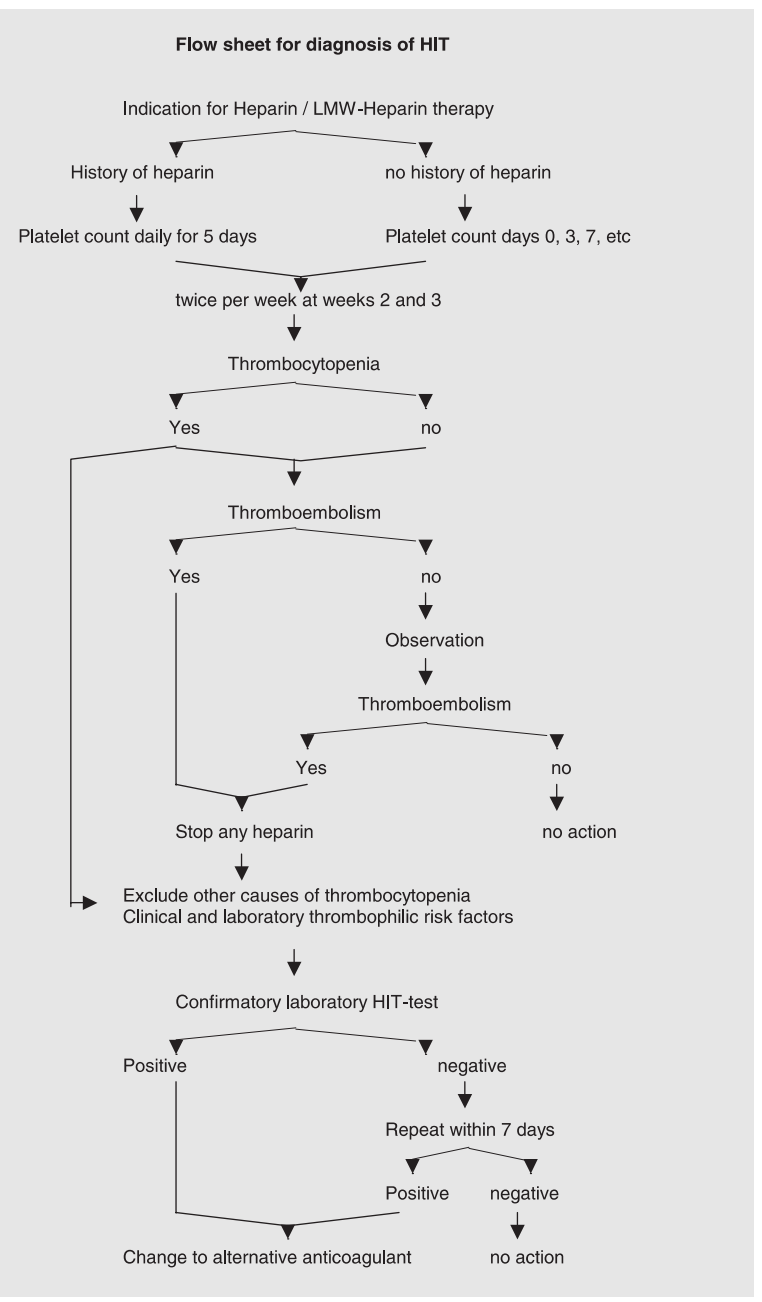

Fig. 2. Decision tree for diagnosis of HIT

interrupt the underlying pathophysiological mechanism. Low sulfated heparinoids or other glycosaminoglycans and direct thrombin inhibitors have been widely adopted to manage HIT type II disease effectively.

Danaparoid, a mixture of low sulfated dermatansulfate and heparansulfate has been successfully used in patients with HIT type II $[13,14]$. The dosage ranges from $3 \times 750$ aXa IU s.c daily to 4.800 IU i.v. daily with a target aXa level between 0.4 and $0.8 \mathrm{IU} / \mathrm{ml}$.

Low sulfated dextran 70 has been successfully used in HIT type II patients using $100 \mathrm{mg}$ the first and $500 \mathrm{ml}$ infusions at days $2-5$ [15].

Dermatansulfate was administered effectively to HIT type II patients. In all 5 patients platelet count increased within a few days and thrombus regressed [16].

r-Hirudin is a direct thrombin inhibitor with the largest experience to treat HIT type II patients. A reduced mortality has been described for patients after changing anticoagulation from heparin to lepirudin [17]. A bolus of $0.4 \mathrm{mg} / \mathrm{kg}$ is followed by

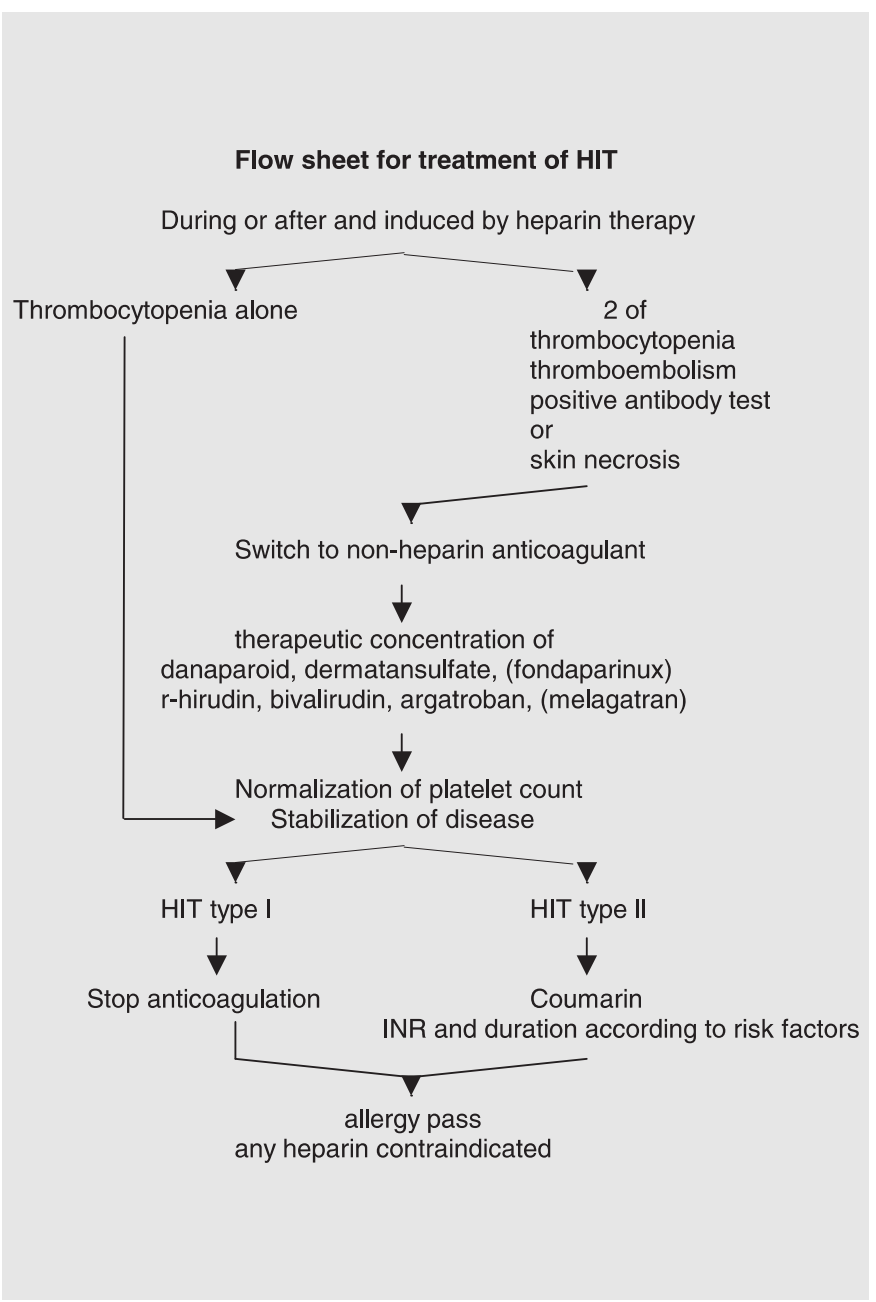

Fig. 3. Decision tree for treatment of patients with HIT

continuos i.v. infusion of $0.4 \mathrm{mg} / \mathrm{kg} / \mathrm{hr}$ to maintain a 2 to 3 -fold prolongation of the aPTT. However, lower doses of 0.1 $\mathrm{mg} / \mathrm{kg} / \mathrm{hr}$ resulted in the desired prolongation of aPTT [18].

Bivalirudin is a synthetic polypeptide, which directly inhibits thrombin by simultaneously binding to its active catalytic site and its substrate recognition site. $1.0 \mathrm{mg} / \mathrm{kg}$ body weight as bolus is followed by $2.5 \mathrm{mg} / \mathrm{kg} / \mathrm{h}$ to maintain 2 to 3 fold prolongation of the aPTT. This successful use of bivalirudin in HIT type II patients has been described in patients undergoing percutaneous coronary interventions [19].

Argatroban is a synthetic low-molecular-weight direct thrombin inhibitor binding to the catalytic side of thrombin. The successful use in HIT type II patients has been described using $2 \mu \mathrm{g} / \mathrm{kg} / \mathrm{min}$ intravenously to maintain the aPTT 1.5 to 3 times the baseline value [20].

Anticoagulation of HIT type II patients has been reported using glycoprotein IIb/IIIa receptor antagonist tirofiban during cardiopulmonary bypass operation of HIT type II patients. However, it has to be considered that anticoagulation was per- 
formed with unfractionated heparin after a bolus of $1 \mu \mathrm{g} / \mathrm{kg}$ tirofiban followed by $0.15 \mu \mathrm{g} / \mathrm{kg} / \mathrm{min}$ until $1 \mathrm{~h}$ before the pulmonary bypass. Further reports and the analysis of the pathophysiological mechanism should be expected before recommendation of this therapy [21].

\section{Long term management of HIT patients}

Cutaneous allergy to heparins should be defined by cutaneous testing since cross-reactions between low-molecularweight heparins as well as heparinoids may be observed [22].

Prophylaxis of recurrent thromboembolism has to be performed with coumarins in all patients with thromboembolic complications for a minimum of 6 months. However, oral anticoagulation should be started after normalisation of platelet counts [18]. Acetylsalicylic acid should be restricted to patients with coronary thrombosis and hemorrhagic transformation of cerebral ischemia caused by HIT type II.

\section{Diagnostic and therapeutic decision trees}

The suspicion of heparin-induced thrombocytopenia is made on the clinical basis only. The symptoms of thrombocytopenia and/or development or progression of thrombosis dur- ing heparinisation are the main symptoms after exclusion of other drug-related side effects. The diagnostic decision tree is given in Figure 2.

Heparin should be switched to an alternative anticoagulant immediately in HIT type II. In patients with a history of HIT type II any heparin is contraindicated and alternative anticoagulants is to be used in any clinical situation, if prophylaxis/treatment of thromboembolism is indicated (Figure 3).

Laboratory confirmation of the clinical suspicion should be performed. Risk factors have to be identified. Anticoagulation should be maintained in HI type I for 6-14 days after stopping anticoagulation with heparins. In HIT type II, antithrombotic therapy should continue for a minimum of 6 months. In the presence of additional risk factors, such as Lupus anticoagulant or permanent risk factors, anticoagulation with coumarins has to be performed even longer.

\section{Perspectives}

Pentasaccharide has been developed as an antithrombotic drug and is effective for prophylaxis and treatment of thromboembolism [23]. Oral direct thrombin inhibitors are effective for prophylaxis and treatment of thromboembolism [24] and may be used in HIT type II patients after approval.

\section{References}

1. Petitou M, Herault JP, Bernat A, Driguez PA, Duchaussoy P, Lormeau JC, Herbert JM: Synthesis of thrombin-inhibiting heparin mimetics without side effects. Nature 1999;398:417-422.

2. Harenberg J, Huhle G, Wang LC, Hoffmann $\mathrm{U}$, Bayerl Ch, Kerowgan M: Association of heparin-induced skin lesions, intracutaneous tests, and heparin-induced IgG. Allergy 1999; 54:473-477.

3. Li ZQ, Liu W, Park KS, Sachais BS, Arepally GM, Cines DB, Poncz M: Defining a second epitope for heparin-induced thrombocytopenia/thrombosis antibodies using KKO, a murine HIT-like monoclonal antibody. Hemost Thromb Vasc Biol 2002; 99: 1230-1236.

4. Carlsson LE, Santoso S, Baurichter G, Kroll H, Papenberg S, Eichler P, Westerdaal NAC, Kiefel V, van de Winkel JGJ, Greinacher A: Heparin-induced thrombocytopenia: New insights into the impact of the Rcg RIIa-RH131 Polymorphism. Blood 1998; 92:15261531.

5. Sheridan D, Carter C, Kelton JG: A diagnostic test for heparin-induced thrombocytopenia. Blood 1986:67:27-30.

6. Harenberg J, Huhle G, Giese Ch, Wang LC, Feuring, M, Song XH, Hoffmann U: Determination of serotonin release from platelets by enzyme immunoassay in the diagnosis of heparin-induced thrombocytopenia.
Br J Haematol 2000;109:182--186.

7. Koch S. Harenberg J, Ödel M, Schmidt-Gayk $\mathrm{H}$, Walch S, Budde U: Development of a highpressure liquid chromatography method for diagnosis of heparin-induced thrombocytopenia. Am J Clin Pathol 2002;117;900-904.

8. Warkentin TE, Sheppard J-A, Horsewood P, Simpson PJ, Moore JC, Kelton JG: Impact of the patient population on the risk for heparininduced thrombocytopenia. Blood 2000;96:1703-1708.

9. Amiral J, Marfaing-Koka A, Wolf M, Alessi MC, Tardy B, Boyer-Neumann C, Vissac AM, Fressinaud E, Poncz M, Meyer D: Presence of autoantibodies to interleukin-8 or neutrophilactivating peptide- 2 in patients with heparinassociated thrombocytopenia. Blood 1996;88:410-416.

10. Harenberg J, Wang LC, Hoffmann U, Huhle G, Feuring M: Laboratory diagnosis of heparin-induced throm-bocytopenia type II after clearance of platelet factor 4/heparin complex. J Lab Clin Med 2001:137:408-413.

11. Warkentin TE, Kelton JG: Temporal aspects of heparin-induced thrombocytopenia. N Engl J Med 2001;344:1286-1292.

12. Harenberg J, Wang LC, Hoffmann U, Huhle G, Feuring M: Improved laboratory confirmation of heparin-induced thrombocytopenia type II. Am J Clin Pathol 2001:115:432-438.

13. Harenberg J, Zimmermann R, Schwarz F,
Kübler W: Treatment of heparin-induced thrombocytopenia with thrombosis by new heparinoid. Lancet 1983;1:986-987.

14. Magnani HN: Heparin-induced thrombocytopenia (HIT): an overview of 230 patients treated with orgaran (Org 10172). Thromb Haemost 1993;70:554-561.

15. Chong BH, Gallus AS, Cade JF, Magnani H, Manoharan A, Oldmeadow M, Arthur C, Rickard K, Gallo J, Lloyd J, Seshadri P, Chesterman CN, The Australian HIT Study Group. Prospective randomised open-label comparison of danaparoid with dextran 70 in the treatment of heparin-induced thrombocytopenia with thrombosis: a clinical outcome study. Thromb Haemost 2001;86:1170-1175.

16. Taliani MR, Agnelli G, Nenci GG, Gianese F: Dermatan sulphate in patients with heparininduced thrombocytopenia. $\mathrm{Br} \mathrm{J}$ Haematol 1999; 104:87-89.

17. Greinacher A, Eichler P, Lubenow N, Kwasny H, Luz M: Heparin-induced thrombocytopenia with thromboembolic complications: metaanalysis of 2 prospective trials to assess the value of parenteral treatment with lepirudin ad its therapeutic aPTT range. Blood 2000;96:846-851.

18. Harenberg J, Huhle G, Piazolo L, Wang LC, Heene DL: Anticoagulation with heparininduced thrombocytopenia type II. Semin Thromb Hemost 1997;23:189-195. 
19. Campbell KR, Mahaffey KW, Lewis BE, Weitz JI, Berkowitz SD, Ohman EM, Califf RM: Bivalirudin in patients with heparininduced thrombocytopenia undergoing percutaneous coronary intervention. J Invasive Cardiol 2000;12 (suppl F):14-19.

20. Lewis BE, Wallis DE, Berkowitz SD, Matthai WH; Fareed J, Walenga JM, Bartholomew J, Sham R, Lerner RG, Zeigler ZR, Rustagi ZR, Rustagi PK, Jang IJ, Rifkin SD, Moran J, Hursting MJ, Kelton JG, ARG-911 Study Investigators. Circulation 2001;103:18381843.
21. Koster A, Kukucka M, Bach F, Meyer O, Fischer T, Mertzlufft F, Loebe M, Hetzer R, Kuppe H: Anticoagulation during cardiopulmonary bypass in patients with heparininduced thrombocytopenia type II and renal impairment using heparin and the platelet glycoprotein IIb/IIIIa antagonist tirofiban. Anesthesiology 2001;94:245-251.

22. Harenberg J, Hoffmann U, Huhle G, Winkler $\mathrm{M}$, Bayerl Ch: Cutaneous reactions to anticoagulants. Am J Clin Dermatol 2001;2: 69-75.

23. Turpie AG, Gallus AS, Hoek JA: A synthetic pentasaccharide for the prevention of deep- vein thrombosis after total hip replacement. $\mathrm{N}$ Engl J Med 2001;344:619-625.

24. Schulman S, Wahlander K, Gustafsson D, Welin L, Frison L, Eriksson H, THRIVE I: Efficacy and tolerability of the novel, oral direct thrombin inhibitor Ximelagatran compared with standard therapy for the treatment of acute deep vein thrombosis. Blood 2001;(suppl):1875(abstr). 